\title{
Antibiotics and antiseptics for pressure ulcers (Protocol)
}

\author{
Norman G, Dumville JC, Moore ZEH, Tanner J, Christie J
}

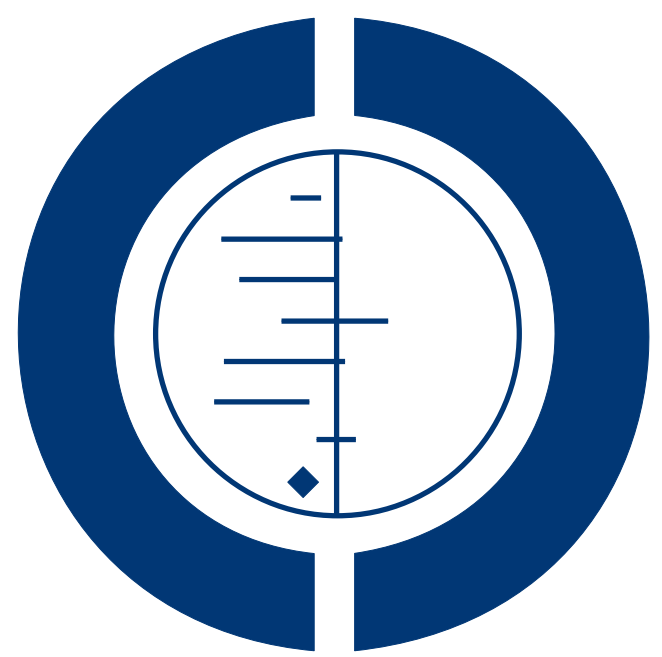

\section{THE COCHRANE COLLABORATION $^{\circledR}$}

This is a reprint of a Cochrane protocol, prepared and maintained by The Cochrane Collaboration and published in The Cochrane Library 2015, Issue 3

http://www.thecochranelibrary.com

\section{WILEY}


TABLE OF CONTENTS

HEADER . . . . . . . . . . . . . . . . . . . . . . . . . . . . . . . . . . . . 1

ABSTRACT . . . . . . . . . . . . . . . . . . . . . . . . . . . . . . . . . . . . . . . . . .

BACKGROUND . . . . . . . . . . . . . . . . . . . . . . . . . . . . . . . . . . . .

OBJECTIVES . . . . . . . . . . . . . . . . . . . . . . . . . . . . . . . . . . . . . . . 5

METHODS . . . . . . . . . . . . . . . . . . . . . . . . . . . . . . . . . . . . . . 5

ACKNOWLEDGEMENTS . . . . . . . . . . . . . . . . . . . . . . . . . . . . . . . . . . . . . . . .

REFERENCES . . . . . . . . . . . . . . . . . . . . . . . . . . . . . . . . . . . . . . 11

APPENDICES . . . . . . . . . . . . . . . . . . . . . . . . . . . . . . . . . . . . . 15

WHAT'S NEW .. . . . . . . . . . . . . . . . . . . . . . . . . . . . . . . . . . . . . . . . . . . . . . . 19

CONTRIBUTIONS OF AUTHORS . . . . . . . . . . . . . . . . . . . . . . . . . . . . . . . . . . . . . 19

DECLARATIONS OF INTEREST . . . . . . . . . . . . . . . . . . . . . . . . . . . . . . . . . . . . . . .

SOURCES OF SUPPORT . . . . . . . . . . . . . . . . . . . . . . . . . . . . . . . . . . . . 


\title{
[Intervention Protocol]
}

\section{Antibiotics and antiseptics for pressure ulcers}

\author{
Gill Norman $^{1}$, Jo C Dumville ${ }^{1}$, Zena EH Moore ${ }^{2}$, Judith Tanner ${ }^{3}$, Janice Christie ${ }^{1}$ \\ ${ }^{1}$ School of Nursing, Midwifery and Social Work, University of Manchester, Manchester, UK. ${ }^{2}$ School of Nursing \& Midwifery, Royal \\ College of Surgeons in Ireland, Dublin, Ireland. ${ }^{3}$ School of Health Sciences, University of Nottingham, Nottingham, UK \\ Contact address: Gill Norman, School of Nursing, Midwifery and Social Work, University of Manchester, Jean McFarlane Building, \\ Oxford Road, Manchester, M13 9PL, UK. gill.norman@manchester.ac.uk.
}

Editorial group: Cochrane Wounds Group.

Publication status and date: Edited (no change to conclusions), published in Issue 3, 2015.

Citation: Norman G, Dumville JC, Moore ZEH, Tanner J, Christie J. Antibiotics and antiseptics for pressure ulcers. Cochrane Database of Systematic Reviews 2015, Issue 3. Art. No.: CD011586. DOI: 10.1002/14651858.CD011586.

Copyright (C) 2015 The Cochrane Collaboration. Published by John Wiley \& Sons, Ltd.

\begin{abstract}
A B S T R A C T
This is the protocol for a review and there is no abstract. The objectives are as follows:

To assess the effects of systemic and topical antimicrobials, and topical antiseptics on the healing of infected and uninfected pressure ulcers being treated in any clinical setting.
\end{abstract}

\section{B A C K G R O U N D}

\section{Description of the condition}

Pressure ulcers, also known as bedsores, decubitus ulcers, pressure injuries or pressure sores are defined as "a localized injury to the skin and/or underlying tissue usually over a bony prominence, as a result of pressure, or pressure in combination with shear" (EPUAP-NPUAP-PPPIA 2014). Pressure ulcers are a type of complex wound that heals by secondary intention (from the bottom up).

Prolonged exposure of an area of the body to pressure or compression can damage cells, interrupt the local blood circulation (i.e. reduce perfusion), and trigger a cascade of biochemical changes that may lead to tissue damage and ulceration (Gebhardt 2002; Loerakker 2010). Immobility can also lead to increased damage from shear and friction, for example, when people are pulled into position in chairs and beds.

People at particular risk of pressure ulcers are those who cannot reposition themselves when they are seated in a chair or lying in bed. This includes those with limited activity and mobility or reduced bodily sensation, such as elderly people, people with spinal cord injuries (Gefen 2014), and those with acute or chronic health conditions (Allman 1997; Bergstrom 1998; Berlowitz 1990; Brandeis 1994). A recent systematic review, Coleman 2013, identified the key risk factors for pressure ulcers as: limitations of mobility or activity; reduced perfusion (including a diagnosis of diabetes); and the presence of a stage 1 pressure ulcer (see classification below). A recent cohort study found that predictors of poor healing included the severity of the ulcer and the presence of peripheral arterial disease (poor circulation/perfusion of the limb; McGinnis 2014).

Children with pressure ulcers are recognised as a discrete population that includes both neonates and older children with a range of conditions and risk factors (EPUAP-NPUAP-PPPIA 2014; NICE 2014); they are cared for in specialist paediatric facilities, and, accordingly, are outside the scope of this review.

\section{Classification of pressure ulcers}


One of the most widely recognised ways of classifying pressure ulcers according to severity is that of the National Pressure Ulcer Advisory Panel (NPUAP). Their international classification recognises four categories, or stages, of pressure ulcers and two categories of unclassifiable pressure injuries in which wound depth or extent, or both, cannot be accurately determined: such ulcers are generally severe and would be grouped clinically with category 3 or 4 ulcers (EPUAP-NPUAP-PPPIA 2014). The definitions for the categories of severity for ulcers are as follows:

Category/Stage 1: non-blanchable erythema: "Intact skin with non-blanchable redness of a localised area usually over a bony prominence. Darkly pigmented skin may not have visible blanching; its colour may differ from the surrounding area. The area may be painful, firm, soft, warmer or cooler as compared to adjacent tissue. Category/Stage I may be difficult to detect in individuals with dark skin tones. May indicate 'at risk' individuals (a heralding sign of risk)."

Category 2: partial thickness tissue loss: "Partial thickness loss of dermis presenting as a shallow open ulcer with a red pink wound bed, without slough. May also present as an intact or open/ruptured serum-filled or sero-sanguinous filled blister. Presents as a shiny or dry shallow ulcer without slough or bruising (bruising indicates suspected deep tissue injury). This category/stage should not be used to describe skin tears, tape burns, perineal dermatitis, maceration or excoriation."

Category 3: full thickness tissue loss: "Full thickness tissue loss. Subcutaneous fat may be visible but bone, tendon or muscle are not exposed. Slough may be present but does not obscure the depth of tissue loss. May include undermining and tunnelling. The depth of a Category/Stage III pressure ulcer varies by anatomical location. The bridge of the nose, ear, occiput and malleolus do not have subcutaneous tissue and Category/Stage III ulcers can be shallow. In contrast, areas of significant adiposity can develop extremely deep Category/Stage III pressure ulcers. Bone/tendon is not visible or directly palpable."

Category 4: full thickness tissue loss with exposed muscle, tendon or bone: "Full thickness tissue loss with exposed bone, tendon or muscle. Slough or eschar may be present in some parts of the wound bed. Often includes undermining and tunnelling. The depth of a Category/Stage IV pressure ulcer varies by anatomical location. The bridge of the nose, ear, occiput and malleolus do not have subcutaneous tissue and these ulcers can be shallow. Category/Stage IV ulcers can extend into muscle and/or supporting structures (e.g., fascia, tendon or joint capsule) making osteomyelitis possible. Exposed bone/muscle is visible or directly palpable." The two additional categories of unclassifiable wounds that are also recognised are:

Unstageable/unclassified: full thickness skin or tissue lossdepth unknown: "Full thickness tissue loss in which actual depth of the ulcer is completely obscured by slough (yellow, tan, gray, green or brown) and/or eschar (tan, brown or black) in the wound bed. Further description: Until enough slough and/or eschar are removed to expose the base of the wound, the true depth cannot be determined; but it will be either a Category/Stage III or IV. Stable (dry, adherent, intact without erythema or fluctuance) eschar on the heels serves as "the body's natural biological) cover" and should not be removed."

Suspected deep tissue injury - depth unknown: "Purple or maroon localized area of discoloured intact skin or blood-filled blister due to damage of underlying soft tissue from pressure and/ or shear. Further description: The area may be preceded by tissue that is painful, firm, mushy, boggy, warmer or cooler as compared to adjacent tissue. Deep tissue injury may be difficult to detect in individuals with dark skin tones. Evolution may include a thin blister over a dark wound bed. The wound may further evolve and become covered by thin eschar. Evolution may be rapid exposing additional layers of tissue even with treatment."

\section{Prevalence}

Pressure ulcers are one of the most common types of complex wound. Prevalence refers to the number of people with a pressure ulcer at a point in time, or during a specific time period (Bonita 2006). Prevalence estimates differ according to the population assessed, the assessment methods used and whether or not category 1 ulcers are included in the estimates.

In the UK, national pressure ulcer data are collected across community and acute settings - although data collection is not yet universal - as part of the National Health Service (NHS) Safety Thermometer initiative (Power 2012). In April 2014, prevalence in NHS patients was $4.6 \%$ (National Safety Thermometer Data 2014). These data represent patients cared for across a range of settings including acute hospital wards, community and residential care and at home. Most patients had category 2 ulcers (3.0\%), with $1.1 \%$ having category 3 and $0.6 \%$ having category $4 \mathrm{ul}-$ cers (category 1 ulcers were not included in the reporting). The point prevalence of pressure ulceration in the total adult population (rather than those currently receiving medical treatment) was recently estimated using a cross-sectional survey undertaken in Leeds, in the UK. The total adult population was 751,485 , and the point prevalence (including grade 1 ulcers) was 0.31 per 1000 (Hall 2014). Pressure ulcer prevalence estimates specifically for community settings have reported rates of 0.77 per 1000 adults in a UK urban area (Stevenson 2013).

Worldwide figures show a range of prevalence for pressure ulcers. Data from the USA showed that incidence of facility-acquired (i.e. hospital-acquired) ulcers ranged from $9.2 \%$ (general cardiac care) to $10.3 \%$ (surgical intensive care unit) of which $3.3 \%$ were severe (category 3/4/unclassifiable; VanGilder 2009). Australian estimates of pressure ulcer prevalence in acute care range from $4.5 \%$ to $27 \%$ (Prentice 2001), while in Japan prevalence across 5000 hospitals was reported as being $4.26 \%$ (Sanada 2008). Lower figures $(1.8 \%)$ were noted in a cross-sectional descriptive study of pressure ulcer prevalence in a teaching hospital in China (Zhao 
2010), though data from a survey of hospital patients across several European countries found an overall prevalence of $10.5 \%$ (Vanderwee 2007). A review of pressure ulcer prevalence across Scandinavia, Iceland and Ireland, found that the mean prevalence in Norway was $17 \%$ (range $4.8 \%$ to $29 \%$ ), $16 \%$ in Ireland (range $4 \%$ to $37 \%$ ), $15 \%$ in Denmark (range $2.2 \%$ to $35.5 \%$ ), $25 \%$ in Sweden (range $0.04 \%$ to $42.7 \%$ ), and $8.9 \%$ in Iceland (single study, no range available) (Moore 2013a)

The prevalence in high risk population groups may be very much higher: a survey of people with a spinal cord injury found a point prevalence of $23 \%$; furthermore, the lifetime risk in this group is estimated to be $70 \%$ (Raghaven 2003).

\section{Cost of pressure ulcers}

The cost of treating pressure ulcers in the UK has been estimated to range from GBP 1214 for a stage 1 ulcer to GBP 14,108 for a stage 4 ulcer (Dealey 2012). These cost estimates may be conservative due to the omission of negative pressure wound therapy from costings, which were updated from a point prior to the widespread use of this therapy; they also do not include precautions required for dealing with antibiotic-resistant infection. The main driver of these increased costs is not ulcer category per se but the increased rate of complications in higher category ulcers and the subsequent increase in time to healing. In the UK, for the year 2000, the total cost for treating pressure ulcers lay between GBP 1.4 billion and GBP 2.1 billion (Bennett 2004).

Pressure ulcers increase length of hospital stay and associated hospital costs (Allman 1999). Figures from the USA suggest that 'pressure ulcer' was noted as a diagnosis for half a million hospital stays in 2006; for adults, the total hospital cost for these stays was USD 11 billion (Russo 2008). Current data on costs from other healthcare systems are hard to identify, but costs to the Australian healthcare system for treating pressure ulceration have been estimated at AUD 285 million annually (Graves 2005). There is also a substantial societal non-health service cost in prolonged sick leave (absence due to being unwell) for people who are in employment when they develop a pressure ulcer (Gorecki 2009).

\section{Impact of pressure ulcers on people}

The impact of pressure ulcers on affected individuals is large. A systematic review found that pressure ulcers had an impact across physical, social and psychological domains as a result of one or more of the following distressing symptoms: pain, exudate and odour, increased care burden, prolonged rehabilitation, requirement for bed-rest, and hospitalisation (Gorecki 2009). The adjusted health-related quality of life of people with pressure ulcers has been shown to be lower than that for comparable individuals without pressure ulcers (Essex 2009). Pressure ulcers may also become infected, and this can give rise to serious systemic (whole body) infections.

\section{Wound infection}

Chronic wounds such as pressure ulcers offer an ideal environment for microbial colonisation: this is especially true for those pressure ulcers that may be particularly exposed to bacterial contamination from faecal material (Bowler 2001). However, most wounds will contain some micro-organisms and this will not necessarily lead to adverse events (WUWHS 2008).

There are several recognised definitions for wound infection (e.g. CDC 2008; WUWHS 2008). Recently there has been a move away from the view that density of bacteria is the key factor (i.e. that a bacterial load greater than $1 \times 10^{5} \mathrm{~g}^{-1}$ is a predictor of infection) towards the view that infection with enough - or specific types of - pathogenic micro-organisms, or both (Bowler 2003; Davies 2007; Madsen 1996; Trengrove 1996), and the possible production of biofilms (Percival 2004; Wolcott 2008), may lead to negative outcomes and potentially delay healing). However, the impact of microbial colonisation on wound healing is not independent of the host response; the ability of the host to provide an adequate immune response is likely to be as critical in determining whether a wound heals as the specifics of the flora in the wound. Regarding wound flora, investigation into the microbiology of pressure ulcers has been limited - one study of the bacteria present in 25 pressure ulcers of different categories found a mean number of 5.8 species when necrotic tissue was present, but only 1.7 species when it was not (Sapico 1986). A more recent prospective cohort study followed 145 patients with category 2 or higher pressure ulcers: $77 \%$ of these people had pressure ulcers containing Staphylococcus aureus, Gram-negative bacilli or both (Braga 2013). The document 'Wound Infection in Clinical Practice - An International Consensus' defines a scenario leading to wound infection where 'bacteria multiply, healing is disrupted and wound tissues are damaged (local infection) "(WUWHS 2008). The document also notes that "Bacteria may produce problems nearby (spreading infection) or cause systemic illness (systemic infection) “. Indeed, wound infection has been conceptualised as being at one end of a continuum of infection (Kingsley 2004).

Kingsley defined a continuum of infection that begins with sterility (a brief period, possibly following surgery) and progresses through contamination (presence of microbes but little active growth and no clinical problems), to colonisation (the normal status quo with wound flora being managed by the host immune system and no damage to wound tissues), culminating in critical colonisation and then infection (Kingsley 2004).

In addition, Kingsley defined critical colonisation as a point between colonisation and infection where the 'healthy' balance of wound flora is no longer maintained by the host, and the bacterial load or species present in the wound, or both, shift away from a so-called safe level (Kingsley 2004). Others have conceptualised critical colonisation as invasion of the wound surface by microorganisms (AWMA 2011; Edwards 2004).

The classic clinical signs of infection include localised pain, heat, redness, swelling and purulence (pus). The concept of critical 
colonisation lacks clear diagnostic criteria; it is generally noted as being associated with delayed healing in the absence of overt signs of wound infection (Carville 2008; Cutting 2004), possibly with other symptoms such as increased exudate (though less than in infection) and hypergranulation/friable tissue (Cutting 2004; Gardner 2001), although associated evidence is limited.

We have been unable to identify recent or large-scale data on the rates of clinical infection of pressure ulcers; early studies of small numbers of patients produced an estimate of 1.4 cases of infection per 1000 patient days with an ulcer (Nicolle 1994), while a point prevalence study found that $6 \%$ of all nursing home residents participating received treatment for an infected pressure ulcer ( Garibaldi 1981).

Although there is a widespread view amongst those with clinical expertise in the field that healing of pressure ulcers is likely to be retarded by critical colonisation or topical/local infection, the empirical evidence to support this is extremely limited (Howell-Jones 2005). Indeed, the Australian Wound Management Association states that "The true extent of bacterial impairment of wound healing is unknown" (AWMA 2011). In particular there is a dearth of clinical studies to demonstrate a link between infection resolution or reduction of the microbiological load and wound healing; to date, randomised evidence has not supported a link between reduction in bacterial load and faster healing in pressure ulcers (Jull 2013; O'Meara 2001; Storm-Versloot 2010). This may stem in part from the difficulty of culturing micro-organisms from the biofilms present in pressure ulcers (Smith 2010), meaning that microbiological load is not accurately represented in samples.

There is a limited and conflicting evidence base for the relationship between bacterial load, or diversity or structure, and wound healing in other types of chronic wounds such as venous leg and diabetic foot ulcers (Davies 2007; Halbert 1992; Hansson 1995; Madsen 1996; Moore 2010; Sotto 2012). The applicability of this evidence to pressure ulcers is uncertain, as there are known microbiological differences between the wound types. In particular the proportion of anaerobic bacteria (thought to be correlated with non-healing) and mycobacteria appears to be higher in pressure ulcers than in venous leg ulcers (Dowd 2008). There are known differences in the microbiology of pressure ulcers at different stages of healing, but no demonstration that these differences are implicated in the healing process (Sapico 1986).

\section{Description of the intervention}

\section{Standard care for pressure ulcers}

Standard care for adults with pressure ulcers includes the use of pressure redistribution devices such as high-specification foam mattresses or cushions, or both (McInnes 2011); debridement where appropriate and non-gauze dressings (BNF 2013), with foam, hydrocolloid or alginate bases (NICE 2014). Other general strategies include the provision of patient education, management of pain, optimising circulation/perfusion, optimising nutrition and, where appropriate, performing surgical wound closure (AWMA 2011; EPUAP-NPUAP-PPPIA 2014). Treatment of clinical infection is also a key strategy as it is thought that a locally infected wound might show retarded healing and may give rise to a systemic infection.

Routine use of antibiotics and antiseptics is not recommended for the treatment of uninfected pressure ulcers in adults, and systemic antibiotics are recommended only when there is clinical evidence of systemic sepsis (serious infection), spreading cellulitis (deep skin infection) or underlying osteomyelitis (bone infection; NICE 2014). Antibiotic use should be restricted to cases of clear clinical need in the treatment of pressure ulcers, as with all conditions. Internationally, antibiotic-resistant bacteria and multidrugresistant bacteria are increasing as a clinical problem; these bacteria have been found in isolates from a substantial proportion of patients with pressure ulcers, even in community settings (Cataldo 2011; Ellis 2003; Heym 2004). Inappropriate use of antibiotics is not restricted to those given systemically; topical antibiotics are also not recommended for use on non-infected wounds (NICE 2014).

There are two main approaches when an antimicrobial intervention is considered clinically appropriate: an antibiotic may be administered systemically (orally, intravenously or intramuscularly), or a topical antibiotic or antiseptic may be applied (NICE 2014). Antibiotics are substances that destroy or inhibit the growth of micro-organisms (Macpherson 2004). Systemic antibiotic treatments include groups of drugs that share similar modes of action such as penicillins, cephalosporins, aminoglycosides, macrolides and quinolones. Other antibiotics that do not belong to one of these main groups include clindamycin, metronidazole, trimethoprim and co-trimazole (BNF 2013).

Topical antimicrobial agents that are applied directly to the ulcer include both antibiotics and antiseptics. Antiseptics are thought to prevent the growth of pathogenic micro-organisms without damaging living tissue (Macpherson 2004). Topical applications broadly fall into lotions used for wound irrigation or cleaning with a brief contact time (unless used as a pack/soak placed into or onto the wound), or both, and products that are in prolonged contact with the wound such as creams, ointments and impregnated dressings (BNF 2013). Agents used primarily for wound irrigation/ cleaning are commonly based on povidone-iodine, chlorhexidine and peroxide agents. Less commonly used agents include traditional products such as gentian violet and hypochlorites. Creams and ointments for longer contact include fusidic acid, mupirocin, neomycin sulphate and iodine (often as cadexomer iodine; BNF 2013).

The British National Formulary (BNF) categorises antimicrobial dressings under honey-based, iodine-based, silver-based and 'other', which includes dressings impregnated with agents such 
as chlorhexidine or peroxides. Recommendations about dressing types are based primarily on the level of wound exudate, as this determines the dressing substrate, as well as the antimicrobial agent (BNF 2013).

Despite guidance from NICE there is a high use of silver dressings (11\%) compared to other antimicrobial dressings (2\% for next most commonly prescribed antimicrobial dressing). High prescription costs mean that silver dressings account for a disproportionate amount $(22 \%)$ of the annual NHS expenditure on dressings (MeReC 2010). It seems possible that some of these dressings are being used prophylactically (i.e. to prevent infection in wounds that are not clinically infected). There is also a high level of use of both systemic antibiotics and topical agents in patients with chronic wounds. General practice morbidity data for Wales from 2000 showed that twice as many patients with chronic wounds were prescribed systemic antibiotics in the previous year compared to matched controls, with a mean number of prescriptions per year of 2.3 (range 0 to 22) compared to 0.6 (range 0 to 14) for control patients. The same data showed high levels of prescription for topical agents such as silver sulfadiazine (185 times per 1000 patients per year) and metronidazole (223 times per 1000 patients per year) in this group (Howell-Jones 2006). Again it appears possible that some prescriptions may be for the treatment of wounds that are not clinically infected.

\section{How the intervention might work}

The rationale for treating clinically infected wounds with antimicrobial and antiseptic agents is to kill or slow the growth of the pathogenic micro-organisms, thus preventing an infection from worsening and spreading (Kingsley 2004). Improved healing may be a secondary benefit, although evidence of an association between wound healing and infection is limited (see Description of the intervention; Jull 2013; O’Meara 2001; Storm-Versloot 2010). There is a widely held view that wounds that do not show clear signs of clinical infection, but have characteristics such as retarded healing, may also benefit from a reduction in bacterial load. Again, evidence for this is limited (see Description of the condition; AWMA 2011, Howell-Jones 2005).

Normally antibiotics work by inhibiting DNA or protein synthesis, or by disrupting bacterial cell walls. Antispectics can be bacteriocidal (in that they kill micro-organisms) or they can work by slowing the growth of organisms (bacteriostatic). Antiseptics can have a wide spectrum of action that is not restricted to bacteria, and often work by damaging the surface of microbes (Macpherson 2004).

\section{Why it is important to do this review}

Whether systemic or topical antimicrobials or topical antiseptics can promote healing in pressure ulcers remains uncertain. An ear- lier systematic review of antimicrobial agents used for the treatment of all types of chronic wounds was not able to generate definitive conclusions about the use of systemic or topical agents in pressure ulcers because of methodological problems in the primary literature (O'Meara 2001). Since the first review was published, a substantial number of additional relevant trials have been published that relate to pressure ulcers; these include trials of silveror honey-based topical preparations. This review is one of a number of Cochrane reviews investigating the use of antibiotics and antiseptics in the treatment of different types of chronic wounds, each of which updates elements of the original O'Meara review (O'Meara 2001). While there will be some overlap with Cochrane reviews of individual antimicrobial agents in wounds (Jull 2013; Storm-Versloot 2010), and with reviews of different types of dressings (Dumville 2014a; Dumville 2014b; Moore 2013b), this review will provide a single synthesis of the randomised evidence relating to all systemic and topical antimicrobials. Two notable systematic reviews of a range of treatments for pressure ulcers have included some types of antibacterial treatments in wider assessments of dressings or topical treatments (Reddy 2008; Smith 2013). A comprehensive review of all antiseptic and antibiotic treatment of pressure ulcers is, however, lacking.

There are a wide range of options available to health professionals who are considering using antimicrobial therapy for pressure ulcers, either as a treatment for or prophylaxis against clinical infection. Evidence-based decision-making on the impact of antimicrobial agents on healing of pressure ulcers can be challenging. Key problems include decisions about whether or when to use an antibacterial agent instead of standard care, and whether different anti-microbial preparations have different impacts on healing.

\section{O B J E C T I VES}

To assess the effects of systemic and topical antimicrobials, and topical antiseptics on the healing of infected and uninfected pressure ulcers being treated in any clinical setting.

\section{METHODS}

\section{Criteria for considering studies for this review}

\section{Types of studies}

Elements of this methods section are based on the standard Cochrane Wounds Protocol Template.

We will include published and unpublished randomised controlled trials (RCTs), including cluster RCTs, irrespective of language of report. We will include cross-over trials only if they report outcome 
data at the end of the first treatment period, prior to cross-over. Quasi-randomised studies will be excluded. We will include RCTs reported only as abstracts only when available data are sufficient for reasonable data extraction either from the abstract itself or from the study authors.

\section{Types of participants}

We will include studies that recruited adults diagnosed with a pressure ulcer of category 2 or above (i.e. worse) managed in any care setting. We will exclude participants with category 1 ulcers. We will accept study authors' definitions of what they classed as a category 2 or above pressure ulcer unless it is clear that wounds with unbroken skin were included. This will include accepting authors' decisions that a wound is a pressure ulcer rather than, for example, an incontinence related sore/wound. Studies that recruited participants with category 2 or above pressure ulcers alongside people with other types of wounds will be included if the results for people with relevant pressure ulcers are presented separately, or are available from the study authors, or if the proportion of participants with pressure ulcers of category 2 or above is at least $75 \%$. We will not restrict the review to trials that recruited only participants with colonised, critically colonised or infected wounds at baseline, but where information about wound status is reported it will be recorded. Unstageable ulcers will be included and will be recorded as such.

\section{Types of interventions}

The primary interventions of interest are topical antiseptic agents or antimicrobial (antibiotic) agents delivered either systemically or topically. We will include any RCT in which the use of a topical or systemic antimicrobial or a topical antiseptic is the only systematic difference between treatment groups. Systemic antibiotics may be administered orally or by other routes (e.g. intravenously, intramuscularly). Both intervention and control regimens could consist of antibiotics or antiseptics administered singly or in combination; control regimens might also include placebo, another therapy, standard care or no treatment. Studies that evaluate intervention schedules that include other therapies (e.g. pressure relieving devices, dressings) will be included, provided that these treatments were delivered in a standardised way across the trial arms. We will exclude trials in which the presence or absence of a specific antibiotic/antiseptic is not the only systematic difference between trial arms.

We will also exclude evaluations of antibiotics/antiseptics used to prepare for the surgical treatment of ulcers (i.e. the surgical closure of ulcers or skin grafting), and physical and biological therapies sometimes purported to have incidental antibacterial properties such as heat therapy and larval therapy.

We anticipate that interventions will consist of antiseptic and antimicrobial agents, which might include (but not be limited to) the following topical agents that may be available in the form of creams, sprays, ointment, or impregnated into different types of dressings: chlorhexidine; povidone-iodine; hydrogen peroxide and potassium permanganate; benzoyl peroxide; hypochlorites (e.g. Eusol); gentian violet; mupirocin and fusidic acid; neomycin sulphate; peroxides; iodine, silver and honey.

Systemic antibiotics might include penicillins, cephalosporins, aminoglycosides, macrolides and quinolones, clindamycin, metronidazole, trimethoprim and co-trimazole.

\section{Types of outcome measures}

We list primary and secondary outcome measures below. If a trial is otherwise eligible (correct study design, population and intervention/comparator) but does not report a relevant listed outcome, then we will contact the study authors where possible in order to establish whether the outcome was measured but not reported.

We will report outcome measures at the latest time point available for a study (assumed to be length of follow-up if not specified) and the time point specified in the methods as being of primary interest (if this is different from latest time point available). For all outcomes we will class (and categorise) outcomes from:

- one to eight weeks as short-term;

- between eight and 26 weeks as medium-term; and

- over 26 weeks as long-term.

Review authors will use their judgement to determine whether statistical pooling within these time categories is appropriate.

\section{Primary outcomes}

The primary effectiveness outcome for this review is wound healing. Trialists use a range of different methods of measuring and reporting this outcome. RCTs that report one or more of the following will be considered to provide the most relevant and rigorous measures of wound healing.

- Time to complete wound healing (correctly analysed using survival, time-to-event approaches). Ideally the outcome will be adjusted for appropriate covariates e.g. baseline ulcer area/ duration.

- Proportion of wounds completely healed during follow-up (frequency of complete healing).

We will use, and report, authors' definitions of complete wound healing. We will report outcome measures at the latest time point available (assumed to be length of follow-up, if not specified) and the time point specified in the methods as being of primary interest (if this is different from latest time point available).

Where both the outcomes above are reported we will present all data in a summary outcome table for reference, but will focus on reporting time to healing. When time is analysed as a continuous measure, but it is not clear whether all wounds healed, we will document the use of the outcome in the study, but we will not extract, summarize or use the data in a meta-analysis. 
The primary safety outcome for the review is all reported adverse events. Reported data will be extracted on all serious and nonserious adverse events when a clear methodology for the collection of adverse event data is provided. This methodology should make it clear whether events were reported at the participant level or whether multiple events/person are reported, in which case appropriate adjustments need to be made for data clustering. Individual types of adverse events other than pain or infection will not be extracted (see Secondary outcomes).

\section{Secondary outcomes}

The following secondary outcomes will be included.

- Change (and rate of change) in wound size, with adjustment for baseline size (we will contact study authors to request adjusted means when not presented). When change or rate of change in wound size is reported without adjustment for baseline size, use of the outcome in the study will be documented, but data will not be extracted, summarised or used in any meta-analysis.

- Changes in infection status; signs or symptoms of clinical infection (we will use study authors' definitions of clinical infection). We will not include data on bacterial load, diversity or the presence of individual species, where it is not clear how the outcome relates to infection.

- Changes in bacterial (antibiotic) resistance.

- Health-related quality of life: quality of life will be included when it is reported using a validated scale such as the SF-36 (Ware 1992) or EQ-5D (EuroQoL Group 1990) or a validated disease-specific questionnaire such as the Cardiff Wound Impact Schedule (Price 2004). Ideally the reported data will be adjusted for the baseline score. We will not include ad hoc measures of quality of life that are unlikely to be validated and would not be common to multiple trials.

- Mean pain scores (including pain at dressing change) will be included only when it is reported as either a presence or absence of pain, or as a continuous outcome using a validated scale such as a visual analogue scale (VAS).

- Resource use (when presented as mean values with standard deviation) including measures such as number of dressing changes, number of nurse visits, length of hospital stay, need for other interventions.

- Costs associated with resource use (including estimates of cost-effectiveness).

\section{Search methods for identification of studies}

\section{Electronic searches}

We will search the following electronic databases:
- The Cochrane Wounds Group Specialised Register (latest version);

- The Cochrane Central Register of Controlled Trials

(CENTRAL; The Cochrane Library; latest issue);

- Ovid MEDLINE (1946 to date);

- Ovid MEDLINE (In-Process \& Other Non-Indexed Citations) latest version;

- Ovid EMBASE (1974 to date);

- EBSCO Cumulative Index to Nursing and Allied Health Literature (CINAHL; 1982 to date).

We will use the following provisional search strategy in The Cochrane Central Register of Controlled Trials (CENTRAL): \#1 MeSH descriptor: [Anti-Infective Agents] explode all trees \#2 MeSH descriptor: [Penicillins] explode all trees \#3 MeSH descriptor: [Cephalosporins] explode all trees \#4 MeSH descriptor: [Aminoglycosides] explode all trees \#5 MeSH descriptor: [Quinolones] explode all trees \#6 MeSH descriptor: [Clindamycin] explode all trees \#7 MeSH descriptor: [Metronidazole] explode all trees \#8 MeSH descriptor: [Trimethoprim] explode all trees \#9 MeSH descriptor: [Mupirocin] explode all trees \#10 MeSH descriptor: [Neomycin] explode all trees \#11 MeSH descriptor: [Fusidic Acid] explode all trees \#12 MeSH descriptor: [Framycetin] explode all trees \#13 MeSH descriptor: [Polymyxins] explode all trees \#14 MeSH descriptor: [Chlortetracycline] explode all trees \#15 (antibiotic* or antimicrobial* or antibacterial* or penicillin* or cephalosporin* or aminoglycoside* or quinolone* or clindamycin or metronidazole or trimethoprim or mupirocin or "pseudomonic acid" or neomycin or "fusidic acid" or framycetin or polymyxin* or chlortetracycline):ti,ab,kw \#16MeSH descriptor: [Antisepsis] explode all trees \#17 antiseptic*:ti,ab,kw

\#18 MeSH descriptor: [Soaps] explode all trees

\#19 MeSH descriptor: [Iodophors] explode all trees \#20 MeSH descriptor: [Chlorhexidine] explode all trees \#21 MeSH descriptor: [Alcohols] explode all trees \#22 MeSH descriptor: [Hydrogen Peroxide] explode all trees \#23 MeSH descriptor: [Benzoyl Peroxide] explode all trees \#24 MeSH descriptor: [Gentian Violet] explode all trees \#25 MeSH descriptor: [Hypochlorous Acid] explode all trees \#26 MeSH descriptor: [Hexachlorophene] explode all trees \#27 MeSH descriptor: [Potassium Permanganate] explode all trees \#28 MeSH descriptor: [Silver] explode all trees \#29 MeSH descriptor: [Silver Sulfadiazine] explode all trees \#30 MeSH descriptor: [Honey] explode all trees \#31 ("soap" or "soaps" or iodophor* or povidone or iodine or chlorhexidine or betadine or "alcohol" or disinfectant* or "hydrogen peroxide" or "benzoyl peroxide" or "gentian violet" or hypochlorit* or eusol or dakin* or hexachlorophene or benzalkonium or "potassium permanganate " or "silver sulfadiazine" or "silver sulphadiazine“ or honey*):ti,ab,kw 
$\# 32 \# 1$ or $\# 2$ or \#3 or \#4 or \#5 or \#6 or \#7 or \#8 or \#9 or \#10 or $\# 11$ or $\# 12$ or $\# 13$ or \#14 or \#15 or \#16 or \#17 or \#18 or \#19 or $\# 20$ or \#21 or \#22 or \#23 or \#24 or \#25 or \#26 or \#27 or \#28 or $\# 29$ or \#30 or \#31

\#33 MeSH descriptor: [Pressure Ulcer] explode all trees

\#34 (pressure next (ulcer* or sore* or injur*)):ti,ab,kw

\#35 (decubitus next (ulcer* or sore*)):ti,ab,kw

\#36 ((bed next sore*) or bedsore):ti,ab,kw

$\# 37$ \#33 or \#34 or \#35 or \#36

\#38 \#32 and \#37

We will combine the Ovid MEDLINE search with the Cochrane Highly Sensitive Search Strategy for identifying randomised trials in MEDLINE: sensitivity- and precision-maximising version (2008 revision; Lefebvre 2011). We will combine the EMBASE search with the Ovid EMBASE filter developed by the UK Cochrane Centre (Lefebvre 2011).

We will combine the CINAHL searches with the trial filters developed by the Scottish Intercollegiate Guidelines Network (SIGN 2011). There will be no restrictions with respect to language, date of publication or study setting.

We will also search the following clinical trials registries:

- ClinicalTrials.gov (http://www.clinicaltrials.gov/);

- WHO International Clinical Trials Registry Platform (http: //apps.who.int/trialsearch/Default.aspx);

- EU Clinical Trials Register (https://

www.clinicaltrialsregister.eu/).

\section{Searching other resources}

We will try to identify other potentially eligible trials or ancillary publications by searching the reference lists of retrieved included trials, as well as relevant systematic reviews, meta-analyses and health technology assessment reports.

\section{Data collection and analysis}

\section{Selection of studies}

Two review authors will independently assess the titles and abstracts of the citations retrieved by the searches for relevance. After this initial assessment, we will obtain full text copies of all studies considered to be potentially relevant. Two review authors will independently check the full papers for eligibility; disagreements will be resolved by discussion and, where required, through the input of a third review author. When the eligibility of a study is unclear we will attempt to contact the study authors. We will record all reasons for exclusion of studies for which we obtained full copies of the text. We will complete a PRISMA flowchart to summarize this process (Liberati 2009).

When studies have been reported in multiple publications/reports, we will obtain all publications. Whilst the study will be included only once in the review, we will extract data from all reports to ensure all available relevant data are obtained.

\section{Data extraction and management}

We will extract and summarize details of the eligible studies. Where possible we will extract data by treatment group for the pre-specified interventions and outcomes in this review. Data will be extracted independently by two review authors; discrepancies will be resolved through discussion or by consultation with a third review author. When data are missing from reports, we will attempt to contact the study authors to request this information.

When a study with more than two intervention arms is included, only data from the intervention and control groups that meet the eligibility criteria will be extracted. When the reported baseline data relate to all participants rather than to those in relevant treatment arms, the data for the whole trial will be extracted and this will be noted. Outcome data will be collected for relevant time points as described in Types of outcome measures.

Where possible we will extract the following data:

- bibliographic data including date of completion/ publication;

- country of origin;

- unit of randomisation (participant/ulcer);

- unit of analysis;

- trial design, e.g. parallel; cluster;

- care setting;

- number of participants randomised to each trial arm and number included in final analysis;

- eligibility criteria and key baseline participant data including category(s) and location(s) of pressure ulcers;

- details of treatment regimen received by each group;

- duration of treatment;

- details of any co-interventions;

- primary and secondary outcome(s) (with definitions and, where applicable, time-points);

- outcome data for primary and secondary outcomes (by group);

- duration of follow-up;

- number of withdrawals (by group) and number of withdrawals (by group) due to adverse events;

- publication status of study;

- source of funding for trial.

\section{Assessment of risk of bias in included studies}

Two review authors will independently assess the included studies using the Cochrane Collaboration tool for assessing risk of bias (Higgins 2011a; Appendix 1). This tool addresses six specific domains: sequence generation, allocation concealment, blinding, incomplete data, selective outcome reporting and other issues in this review we will record issues with unit of analysis, for example where a cluster trial has been undertaken but analysed at 
the individual level in the study report. We will assess blinding of outcome assessment and completeness of outcome data for each of the review outcomes separately. We will present our assessment of risk of bias using two 'Risk of bias' summary figures; one that summarises bias for each item across all studies, and a second that shows a cross-tabulation of each trial by all of the 'Risk of bias' items. We will class studies judged to be at a high risk of bias for either the randomisation sequence domain or the allocation concealment domain or the blinded outcome assessment domain for wound healing - or a combination of these - as having an overall high risk of bias for wound healing (because wound healing is a subjective outcome, there is a high risk of measurement bias when outcome assessment is not blinded). For trials using cluster randomisation, we will also consider the risk of bias considering: recruitment bias, baseline imbalance, loss of clusters, incorrect analysis and comparability with individually randomised trials (Higgins 2011b; Appendix 2).

\section{Measures of treatment effect}

Time-to-event data (e.g. time-to-complete wound healing) will be reported as hazard ratios (HRs) when possible, in accordance with the methods described in the Cochrane Handbook for Systematic Reviews of Interventions (Deeks 2011). If studies reporting timeto-event data (e.g. time to healing) do not report a hazard ratio, then, where feasible, we plan to estimate this using other reported outcomes, such as numbers of events, through the application of available statistical methods (Parmar 1998; Tierney 2007). For dichotomous outcomes we will calculate the risk ratio (RR) with 95\% confidence intervals (CIs). For continuous outcome data, we will use the mean difference (MD) with 95\% CIs for trials that use the same assessment scale, and, when trials use different assessment scales, we will use the standardised mean difference (SMD) with 95\% CIs.

\section{Unit of analysis issues}

Unit of analysis issues may arise with studies that include participants with multiple wounds, who are treated with the same intervention, with outcomes reported for each wound, or with studies in which multiple assessments of an outcome are presented for participants. We will record whether trials presented outcomes in relation to a wound or a participant, or as multiple wounds on the same participant. For wound healing, unless otherwise stated, when the number of wounds appears to equal the number of participants, we will treat the wound as the unit of analysis.

When a cluster-randomised trial has been conducted and analysed correctly, we may meta-analyse effect estimates and their standard errors using the generic inverse variance method in Review Manager 5.3 (RevMan). When a cluster-randomised trial has been conducted, but incorrectly analysed at the individual rather than the cluster level, we will approximate the correct analyses if possible, in accordance with Chapter 16 of the Cochrane Handbook for
Systematic Reviews of Interventions, using information provided in Higgins 2011b.

We will also note when randomisation has been undertaken at the wound level - a split-site or split body design. We will assess whether the correct paired analysis has been undertaken in the study. Again issues will be recorded in the risk of bias section. We will also record occasions when multiple wounds on a participant are (incorrectly) treated in the included study as though they are independent of each other, rather than with application of withinpatient analysis methods. This will be recorded as part of the 'Risk of bias' assessment. If an incorrect analysis has been undertaken we will try to approximate a correct analysis if the required data are available from the study report or the study authors. If this is not possible, we will extract and present the relevant outcome data, but not analyse them further.

\section{Dealing with missing data}

It is common to have data missing from trial reports. The exclusion of participants from the analysis post randomisation or ignoring those lost to follow-up compromises the randomisation and potentially introduces bias into the trial. If we think that study authors might be able to provide some missing data, we will contact them, however, it is likely that data will often be missing because of loss to follow-up. In individual studies, when data are presented for the proportion of ulcers healed, we plan to assume that randomly assigned participants who were not included in the analysis had an unhealed wound at the end of the follow-up period (i.e. they will be considered in the denominator but not in the numerator). When a trial does not specify participant group numbers before dropout, we will present only complete case data. For time-tohealing analysis using survival analysis methods, dropouts should be accounted for as censored data. Hence all participants will contribute to the analysis. We acknowledge that such analysis assumes that dropouts are missing at random and that there is no pattern of missingness. We will present data for area change of ulcer and for all secondary outcomes as complete case analyses.

We will present available data from the study reports/study authors for continuous variables - for example length of hospital stay - and for all secondary outcomes, and do not plan to impute missing data. Where measures of variance are missing we will calculate these wherever possible (Higgins 2011a), where this is not possible we will contact study authors. When these measures of variation remain unavailable and cannot be calculated the study will be excluded from any relevant meta-analyses we conduct.

\section{Assessment of heterogeneity}

Assessment of heterogeneity can be a complex, multi-faceted process. Firstly, we will consider clinical and methodological heterogeneity, that is the degree to which the included studies vary in terms of participant, intervention, outcome and characteristics 
such as length of follow-up. We will supplement this assessment of clinical and methodological heterogeneity with information regarding statistical heterogeneity - we will assess this using the $\mathrm{Chi}^{2}$ test (we will consider that $P$ values less than 0.10 indicate statistically significant heterogeneity) in conjunction with the $\mathrm{I}^{2}$ measure (Higgins 2003). $\mathrm{I}^{2}$ examines the percentage of total variation across RCTs that is due to heterogeneity rather than chance (Higgins 2003). Very broadly we will consider that $\mathrm{I}^{2}$ values of $25 \%$, or less, may mean a low level of heterogeneity (Higgins 2003), and values of more than $75 \%$, or more, indicate very high heterogeneity (Deeks 2011). Where there is evidence of high heterogeneity we will attempt to explore this further: see Data synthesis.

\section{Assessment of reporting biases}

Reporting biases arise when the dissemination of research findings is influenced by the nature and direction of results. Publication bias is one of a number of possible causes of 'small study effects', that is, a tendency for estimates of the intervention effect to be more beneficial in smaller RCTs. Funnel plots allow a visual assessment of whether small study effects may be present in a metaanalysis. A funnel plot is a simple scatter plot of the intervention effect estimates from individual RCTs against some measure of each trial's size or precision (Sterne 2011). Funnel plots are only informative when there are a substantial number of studies included in an analysis; we plan to present funnel plots for metaanalyses that include at least 10 RCTs using RevMan 5.3.

\section{Data synthesis}

We will combine details of included studies in a narrative review according to the comparison between intervention and comparator, the population and the time point of the outcome measurement. We will consider clinical and methodological heterogeneity and undertake pooling when studies appear appropriately similar in terms of ulcer category, intervention type and antibacterial agent, duration of treatment and outcome assessment.

In terms of our meta-analytical approach, in the presence of clinical heterogeneity (review author judgement) or evidence of statistical heterogeneity, or both, we will use the random-effects model. We will only use a fixed-effect approach when clinical heterogeneity is thought to be minimal and statistical heterogeneity is estimated as non-statistically significant for the $\mathrm{Chi}^{2}$ value and $0 \%$ for the $\mathrm{I}^{2}$ assessment (Kontopantelis 2013). We will adopt this approach as it is recognised that statistical assessments can miss potentially important between-study heterogeneity in small samples, hence the preference for the more conservative random-effects model ( Kontopantelis 2012). Where clinical heterogeneity is thought to be acceptable or of interest we may meta-analyse even when statistical heterogeneity is high but we will attempt to interpret the causes behind this heterogeneity and will consider using meta-regression for that purpose, if possible (Thompson 1999; Thompson 2002).
We will present data using forest plots where possible. For dichotomous outcomes we will present the summary estimate as a risk ratio (RR) with $95 \%$ CI. Where continuous outcomes are measured in the same way across studies, we plan to present a pooled mean difference (MD) with $95 \% \mathrm{CI}$; we plan to pool standardised mean difference (SMD) estimates where studies measured the same outcome using different methods. For time to event data, we plan to plot (and, if appropriate, pool) estimates of hazard ratios and $95 \%$ CIs as presented in the study reports using the generic inverse variance method in RevMan 5.3. Where time to healing is analysed as a continuous measure but it is not clear if all wounds healed, use of the outcome in the study will be documented, but those data will not be summarised or used in any meta-analysis.

\section{'Summary of findings' tables}

We will present the main results of the review in 'Summary of findings' tables. These tables present key information concerning the quality of the evidence, the magnitude of the effects of the interventions examined, and the sum of available data for the main outcomes (Schunemann 2011a). The 'Summary of findings' tables also include an overall grading of the evidence related to each of the main outcomes using the GRADE (Grades of Recommendation, Assessment, Development and Evaluation) approach. The GRADE approach defines the quality of a body of evidence as the extent to which one can be confident that an estimate of effect or association is close to the true quantity of specific interest. The quality of a body of evidence involves consideration of within trial risk of bias (methodological quality), directness of evidence, heterogeneity, precision of effect estimates and risk of publication bias (Schunemann 2011b).We plan to present the following outcomes in the 'Summary of findings' tables.

- Time to complete ulcer healing when analysed using appropriate survival analysis methods.

- Proportion of ulcers completely healing during the trial period.

- Changes in clinical infection status.

- Adverse events.

\section{Subgroup analysis and investigation of heterogeneity}

When possible, we will perform subgroup analyses to explore the influence of ulcer category on effect size. If there are sufficient data these analyses will assess whether there are differences in effect sizes for grade 2 pressure ulcers and the more severe grade 3 and 4 (and unclassifiable) pressure ulcers.

When possible, we will perform subgroup analyses to explore the influence of risk of bias on effect size. We will assess the influence of removing studies classed as having high and unclear risk of bias from the meta-analyses. These analyses will include only studies that are assessed as having low risk of bias in all key domains, namely, adequate generation of the randomisation sequence, ad- 
equate allocation concealment and blinding of outcome assessor for the estimates of treatment effect.

\section{ACKNOWLEDGEMENTS}

This work was supported by a grant from National Institute for Health Research, UK (NIHR Cochrane Programme Grant 13/ 89/08-High Priority Cochrane Reviews in Wound Prevention and Treatment).

The authors would like to acknowledge the contribution of the peer referees: Una Adderley; Janet Cuddigan; Marina Israel; Linda Faye Lehman and Liz McInnes. The copy editor: Elizabeth Royle

\section{R E F E R E N C E S}

\section{Additional references}

\section{Allman 1997}

Allman RM. Pressure ulcer prevalence, incidence, risk factors, and impact. Clinical Geriatric Medicine 1997;13(3): 421-36.

\section{Allman 1999}

Allman RM, Goode PS, Burst N, Bartolucci AA, Thomas DR. Pressure ulcers, hospital complications, and disease severity: impact on hospital costs and length of stay. Advances in Wound Care 1999;12:22-30.

\section{AWMA 2011}

Australian Wound Management Association Inc. Position document of the Australian Wound Management Association: Bacterial impact on wound healing: from contamination to infection. www.awma.com.au. Accessed October 2014 2011; Vol. Version 1.5.

\section{Bennett 2004}

Bennett G, Dealey C, Posnett J. The cost of pressure ulcers in the UK. Age and Ageing 2004;33(3):230-5.

\section{Bergstrom 1998}

Bergstrom N, Braden B, Champagne M, Kemp M, Ruby E. Predicting pressure ulcer risk: a multisite study of the predictive validity of the Braden Scale. Nursing Research 1998;47(5):261-9.

\section{Berlowitz 1990}

Berlowitz DR, Wilikin SVB. Risk factors for pressure sores: a comparison of cross-sectional and cohort derived data. Journal of the American Geriatrics Society 1990;37(11): 1043-50.

BNF 2013

British National Formulary, British Medical Association, British Royal Pharmaceutical Society of Great Britain. British National Formulary 66. London: British Medical Association, 2013.

\section{Bonita 2006}

Bonita R, Beaglehole R, Kjellstrom T. Basic Epidemiology. Second Edition. Geneva: World Health Organisation, 2006.

\section{Bowler 2001}

Bowler PG, Duerden BI, Armstrong DG. Wound microbiology and associated approaches to wound management. Clinical Microbiology Reviews 2001;14(2): 244-69.

\section{Bowler 2003}

Bowler PG. The 105 bacterial growth guideline: reassessing its clinical relevance in wound healing. Ostomy Wound Management 2003;49(1):44-53.

\section{Braga 2013}

Braga IA, Pirett CC, Ribas RM, Gontijo Filho PP, Dioggio Filho A. Bacterial colonization of pressure ulcers: assessment of risk for bloodstream infection and impact on patient outcomes.. Journal of Hospital Infection 2013;83(4):314-20.

\section{Brandeis 1994}

Brandeis GH, Ooi WL, Hossain M, Morris JN, Lipsitz LA. A longitudinal study of risk factors associated with the formation of pressure ulcers in nursing homes. Journal of the American Geriatric Society 1994;42:388-93.

\section{Carville 2008}

Carville K, Cuddigan J, Fletcher J, Fuchs P, Harding K, Ishikawa $\mathrm{O}$, et al. Wound infection in clinical practice: shaping the future. An International Consensus Document. International Wound Journal 2008;5(S3):1-11.

\section{Cataldo 2011}

Cataldo MC, Bonura C, Caputo G, Aleo A, Rizzo G, Geraci $\mathrm{D}$, et al. Colonization of pressure ulcers by multidrugresistant microorganisms in patients receiving home care. Scandinavian Journal of Infectious Diseases 2011;43:947-52.

\section{CDC 2008}

Horan TC, Andrus M, Dudeck MA. CDC/NHSN surveillance definition of health care-associated infection 
and criteria for specific types of infections in the acute care setting. American Journal of Infection Control 2008;36: 309-32.

Coleman 2013

Coleman S, Gorecki C, Nelson EA, Defloor T, Halfens R, Farrin A, et al. Patient risk factors for pressure ulcer development: systematic review. International Journal of Nursing Studies 2013;50(7):974-1003.

\section{Cutting 2004}

Cutting KF, White R. Defined and refined: criteria for identifying wound infection revisited. British Journal of Community Nursing 2004;9(3):S6-S15.

\section{Davies 2007}

Davies CE, Hill KE, Newcombe RG, Stephens P, Wilson MJ, Harding KG, et al. A prospective study of the microbiology of chronic venous leg ulcers to reevaluate the clinical predictive value of tissue biopsies and swabs. Wound Repair and Regeneration 2007;15(1):17-22.

Dealey 2012

Dealey C, Posnett J, Walker A. The cost of pressure ulcers in the United Kingdom. Journal of Wound Care 2012;21: 261-2, 264, 266.

Deeks 2011

Deeks JJ, Higgins JPT, Altman DG (editors). Chapter 9: Analysing data and undertaking meta-analyses. In: Higgins JPT, Green S (editors). Cochrane Handbook for Systematic Reviews of Interventions. 5.1.0 (updated March 2011). The Cochrane Collaboration, 2011. Available from www.cochrane-handbook.org.

Dowd 2008

Dowd SE, Sun Y, Secor PR, Rhoads DD, Wolcott BM, James GA, et al. Survey of bacterial diversity in chronic wounds using pyrosequencing, DGGE, and full ribosome shotgun sequencing. BMC Microbiology 2008;8:43. [DOI: $10.1186 / 1471-2180-8-43]$

\section{Dumville 2014a}

Dumville JC, Keogh SJ, Stubbs N, Walker RM. Alginate dressings for treating pressure ulcers. Cochrane Database of Systematic Reviews 2014, Issue 8. [DOI: 10.1002/ 14651858.CD011277]

\section{Dumville 2014b}

Dumville JC, Stubbs N, Keogh SJ, Walker RM. Hydrogel dressings for treating pressure ulcers. Cochrane Database of Systematic Reviews 2014, Issue 7. [DOI: 10.1002/ 14651858.CD011226]

Edwards 2004

Edwards R, Harding KG. Bacteria and wound healing. Current Opinion in Infectious Disease 2004;17:91-6.

\section{Ellis 2003}

Ellis SL, Finn P, Noone M, Leaper DJ. Eradication of methicillin-resistant Staphylococcus aureus from pressure sores using warming therapy. Surgical Infections 2003;4(1): 53-5.

\section{EPUAP-NPUAP-PPPIA 2014}

National Pressure Ulcer Advisory Panel, European Pressure Ulcer Advisory Panel and Pan Pacific Pressure Injury
Alliance. Haesler E (ed). Prevention and Treatment of Pressure Ulcers: Quick Reference Guide. Second Edition. Perth, Australia: Cambridge Media, 2014.

\section{Essex 2009}

Essex HN, Clark M, Sims J, Warriner A, Cullum N. Health-related quality of life in hospital inpatients with pressure ulceration: assessment using generic health-related quality of life measures. Wound Repair Regeneration 2009; 17:797-805.

\section{EuroQoL Group 1990}

EuroQuoL Group. EuroQoL: a new facility for the measurement of health-related quality of life. Health Policy 1990;16:199-208.

\section{Gardner 2001}

Gardner SE, Franz RA, Doebbeling BN. The validity of the clinical signs and symptoms used to identify localized chronic wound infection. Wound Repair and Regeneration 2001;9(3):178-86.

\section{Garibaldi 1981}

Garibaldi RA, Brodine S, Matsumiya S. Infections among patients in nursing homes: policies, prevalence, problems. New England Journal of Medicine 1981;305:731-5.

\section{Gebhardt 2002}

Gebhardt K. Pressure ulcer prevention. Part 1. Causes of pressure ulcers. Nursing Times 2002;98:41-4.

\section{Gefen 2014}

Gefen A. Tissue changes in patients following spinal cord injury and implications for wheelchair cushions and tissue loading: a literature review. Ostomy Wound Management 2014;60:34-45.

\section{Gorecki 2009}

Gorecki C, Brown JM, Nelson EA, Briggs M, Schoonhoven L, Dealey C, et al. Impact of pressure ulcers on quality of life in older patients: a systematic review. Journal of the American Geriatrics Society 2009;57(7):1175-83.

\section{Graves 2005}

Graves N, Birrell F, Whitby M. Modelling the economic losses from pressure ulcers among hospitalized patients in Australia. Wound Repair and Regeneration 2005;13:462-7.

\section{Hahn 2005}

Hahn S, Puffer S, Torgerson DJ, Watson J. Methodological bias in cluster randomised trials. BMC Medical Research Methodology 2005;5:10. [DOI: 10.1186/1471-2288-5-10]

\section{Halbert 1992}

Halbert AR, Stacey MC, Rohr JB, Jopp-McKay A. The effect of bacterial colonization on venous leg ulcer healing. American Journal of Dermatology 1992;33:75-80.

Hall 2014

Hall J, Buckley HL, Lamb KA, Stubbs N, Saramago P, Dumville JC, et al. Point prevalence of complex wounds in a defined United Kingdom population. Wound Repair and Regeneration in press. 


\section{Hansson 1995}

Hansson C, Hoborn J, Moller A, Swanbeck G. The microbial flora in venous leg ulcers without clinical signs of infection. Acta Dermato-venerologica 1995;75(1):24-30.

Heym 2004

Heym B, Rimareix F, Lortat-Jacob A, Nicolas-Chanoine M$\mathrm{H}$. Bacteriological investigation of infected pressure ulcers in spinal cord-injured patients and impact on antibiotic therapy. Spinal Cord 2004;42:230-4.

\section{Higgins 2003}

Higgins JPT, Thompson SG, Deeks JJ, Altman DG. Measuring inconsistency in meta-analyses. BMJ 2003;327 (7414):557-60

\section{Higgins 2011a}

Higgins JPT, Altman DG, Sterne, JAC (editors). Chapter 8: Assessing risk of bias in included studies. In: Higgins JPT, Green S (editors). Cochrane Handbook for Systematic Reviews of Interventions Version 5.1.0 (updated March 2011). The Cochrane Collaboration, 2011. Available from www.cochrane-handbook.org.

\section{Higgins 2011b}

Higgins JPT, Deeks JJ, Altman DG (editors). Chapter 16: Special topics in statistics. In: Higgins JPT, Green S (editors). Cochrane Handbook for Systematic Reviews of Interventions. Version 5.1.0 (updated March 2011). The Cochrane Collaboration, 2011. Available from www.cochrane-handbook.org.

\section{Howell-Jones 2005}

Howell-Hones RS, Wilson MJ, Hill KE, Price PE, Thomas DW. A review of the microbiology, antibiotic usage and resistance in chronic skin wounds. Journal of Antimicrobial Chemotherapy 2005;55(2):143-9.

\section{Howell-Jones 2006}

Howell-Jones RS, Price PE, Howard AJ, Thomas DW. Antibiotic prescribing for chronic skin wounds in primary care. Wound Repair and Regeneration 2006;14:387-93.

\section{Jull 2013}

Jull AB, Walker N, Deshpande S. Honey as a topical treatment for wounds. Cochrane Database of Systematic Reviews 2013, Issue 2. [DOI: 10.1002/ 14651858.CD005083.pub3]

\section{Kingsley 2004}

Kingsley A, White R, Gray D. The wound infection continuum: a revised perspective. APW Supplement. Wounds UK 2004;1(1):13-8.

\section{Kontopantelis 2012}

Kontopantelis E, Reeves D. Performance of statistical methods for meta-analysis when true study effects are nonnormally distributed: a simulation study. Statistical Methods in Medical Research 2012;21(4):409-26.

\section{Kontopantelis 2013}

Kontopantelis E, Springate DA, Reeves D. A re-analysis of the Cochrane Library data: the dangers of unobserved heterogeneity in meta-analyses. PLoS One 2013;26:e69930.

\section{Lefebvre 2011}

Lefebvre C, Manheimer E, Glanville J. Chapter 6: Searching for studies. In: Higgins JPT, Green S (editors). Cochrane Handbook for Systematic Reviews of Interventions. 5.1.0 (updated March 2011). The Cochrane Collaboration, 2011. Available from www.cochrane-handbook.org.

\section{Liberati 2009}

Liberati A, Altman DG, Tetzlaff J, Mulrow C, Gotzsche PC, Ioannidis JP, et al. The PRISMA statement for reporting systematic reviews and meta-analyses of studies that evaluate health care interventions: explanation and elaboration. PLoS Medicine 2009;6:e1000100.

Loerakker 2010 Loerakker S, Stekelenburg A, Strijkers GJ, Rijpkema JJM, Baaijens FPT, Bader DL, et al. Temporal effects of mechanical loading on deformation-induced damage in skeletal muscle tissue. Annals of Biomedical Engineering 2010;38(8):2577-87.

\section{Macpherson 2004} Macpherson G (ed). Black's Student Medical Dictionary. London: A\&C Black Publishers Limited, 2004.

\section{Madsen 1996}

Madsen SM, Westh H, Danielsen L, Rosdahl VT. Bacterial colonisation and healing of venous leg ulcers. APMIS: acta pathologica, microbiologica, et immunologica Scandinavica 1996;104(2):895-9.

\section{McGinnis 2014}

McGinnis E, Greenwood DC, Nelson EA, Nixon J. A prospective cohort study of prognostic factors for the healing of heel pressure ulcers. Age and Aging 2014;43(2): 267-71.

\section{McInnes 2011}

McInnes E, Dumville JC, Jammali-Blasi A, Bell-Syer SE. Support surfaces for treating pressure ulcers. Cochrane Database of Systematic Reviews 2011, Issue 12. [DOI: 10.1002/14651858.CD009490]

MeReC 2010

MeReC. Evidence-based prescribing of advanced wound dressings for chronic wounds in primary care. $\mathrm{MeReC}$ Bulletin 2010; Vol. 21, issue 01.

Moore 2010

Moore K, Hall V, Paull A, Morris T, Brown S, McCullock $\mathrm{D}$, et al. Surface bacteriology of venous leg ulcers and healing outcome. Journal of Clinical Pathology 2010;63: $830-4$.

Moore 2013a

Moore Z, Johansen E, van Etten M. A review of PU prevalence and incidence across Scandinavia, Iceland and Ireland (Part I). Journal of Wound Care 2013;22(7):1-7.

Moore 2013b Moore ZEH, Cowman S. Wound cleansing for pressure ulcers. Cochrane Database of Systematic Reviews 2013, Issue 3. [DOI: 10.1002/14651858.CD004983.pub3]

\section{National Safety Thermometer Data 2014}

National Health Service. National Safety Thermometer Data. http://www.safetythermometer.nhs.uk/index.php? 
option=com_dashboards $\&$ view=classic $\&$ Itemid $=126$

(accessed 4 March 2014).

\section{NICE 2014}

National Institute of Health and Clinical Excellence. Pressure ulcers: prevention and management of pressure ulcers. NICE clinical guideline 179 April 2014:http:/ /www.nice.org.uk/guidance/cg179/resources/guidancepressure-ulcers-prevention-and-management-of-pressureulcers-pdf. Accessed October 2014.

\section{Nicolle 1994}

Nicolle LE, Orr P, Duckworth H, Brunka J, Kennedy J, Urias B, et al. Prospective study of decubitus ulcers in two long term care facilities. Canadian Journal of Infection Control 1994;9(2):35-8.

\section{O'Meara 2001}

O'Meara S, Cullum NA, Majid M, Sheldon TA. Systematic review of antimicrobial agents used for chronic wounds. British Journal of Surgey 2011;88(1):4-21.

\section{Parmar 1998}

Parmar MKB, Torri V, Stewart L. Extracting summary statistics to perform meta-analyses of the published literature for survival endpoints.. Statistics in Medicine 1998;17: 2815-34.

\section{Percival 2004}

Percival SL, Bowler PG. Biofilms and their potential role in wound healing. Wounds 2004;16(7):234-40.

Power 2012

Power M, Stewart K, Brotherton A. What is the NHS Safety Thermometer?. Clinical Risk 2012;18:163-9.

\section{Prentice 2001}

Prentice J L, Stacey M C. Pressure ulcers: the case for improving prevention and management in Australian health care settings. Primary Intention 2001;9:111-20.

\section{Price 2004}

Price, Harding K. Cardiff Wound Impact Schedule: the development of a condition-specific questionnaire to assess health-related quality of life in patients with chronic wounds of the lower limb. International Wound Journal 2004;1(1): $10-17$.

\section{Raghaven 2003}

Raghaven P, Raza WA, Ahmed YS, Chamberlain MA. Prevalence of pressure sores in a community sample of spinal injury patients. Rehabilitation 2003;17(8):879-84.

Reddy 2008

Reddy M, Gill SS, Kalkar SR, Wu W, Anderson PJ, Rochon PA. Treatment of pressure ulcers: a systematic review. JAMA 2008;300:2647-62.

\section{RevMan}

The Nordic Cochrane Centre. Review Manager (RevMan) 5.3. 5.3. Copenhagen: The Nordic Cochrane Centre, 2014.

\section{Russo 2008}

Russo A, Steiner C, Spector W. Hospitalizations related to pressure ulcers among adults 18 years and older, 2006. Healthcare Cost and Utilization Project (HCUP). Acessed 6th June 2014 2008:http://www.hcup-us.ahrq.gov/reports/ statbriefs/sb64.jsp (accessed 6 June 2014).

\section{Sanada 2008}

Sanada H, Miyachi Y, Ohura T, Moriguchi T, Tokunaga K, Shido K, et al. The Japanese pressure ulcer surveillance study: a retrospective cohort study to determine prevalence of pressure ulcers in Japan. Wounds 2008;20(7):20-6.

Sapico 1986

Sapico FL, Ginunas VJ, Thornhill-Jones M, Canawati HN, Klein NE, Khawam S, et al. Quantitative microbiology of pressure sores in different stages of healing. Diagnostic Microbiology and Infectious Disease 1986;5(1):31-8.

\section{Schunemann 2011a}

Schünemann HJ, Oxman AD, Higgins JPT, Vist GE, Glasziou P, Guyatt GH. Chapter 11: Presenting results and 'Summary of findings' tables. In: Higgins JPT, Green S (editors). Cochrane Handbook for Systematic Reviews of Interventions Version 5.1.0 (updated March 2011). The Cochrane Collaboration, 2011. Available from www.cochrane-handbook.org.

\section{Schunemann 2011b}

Schünemann HJ, Oxman AD, Higgins JPT, Deeks JJ, Glasziou P, Guyatt GH. Chapter 12: Interpreting results and drawing conclusions. In: Higgins JPT, Green S (editors). Cochrane Handbook for Systematic Reviews of Interventions Version 5.1.0 (updated March 2011). The Cochrane Collaboration, 2011. Available from www.cochrane-handbook.org.

\section{SIGN 2011}

Scottish Intercollegiate Guidelines Network (SIGN). Search filters. http://www.sign.ac.uk/methodology/filters.html\# random (accessed December 2011).

\section{Smith 2010}

Smith DM, Snow DE, Rees E, Zischkau Am, Hanson JD, Wolcott RD, et al. Evaluation of the bacterial diversity of pressure ulcers using bTEFAP pyrosequencing. BMC Medical Genomics 2010;3:41. [DOI: 10.1186/ 1755-8794-3-41]

\section{Smith 2013}

Smith ME, Totten A, Hickam DH, Fu R, Wasson N, Rahman B, et al. Pressure ulcer treatment strategies: a systematic comparative effectiveness review. Annals of Internal Medicine 2013;159:39-50.

\section{Sotto 2012}

Sotto A, Richard J-L, Messad N, Molinari N, Jourdan $\mathrm{N}$, Schuldiner $\mathrm{S}$, et al. Distinguishing colonization from infection with Staphylococcus aureus in diabetic foot ulcers with miniaturized oligonucleotide arrays: a French multicenter study. Diabetes Care 2012;35:617-23.

\section{Sterne 2011}

Sterne JAC, Egger M, Moher D. Chapter 10: Addressing reporting biases. In: Higgins JPT, Green S (editors). Cochrane Handbook for Systematic Reviews of Interventions Version 5.1.0 (updated March 2011). The Cochrane Collaboration, 2011. Available from www.cochrane-handbook.org. 


\section{Stevenson 2013}

Stevenson R, Collinson M, Henderson V, Wilson L, Dealey $\mathrm{C}, \mathrm{McGinnis} \mathrm{E}$, et al. The prevalence of pressure ulcers in community settings: an observational study. International Journal of Nursing Studies 2013;50:1550-7.

Storm-Versloot 2010

Storm-Versloot MN, Vos CG, Ubbink DT, Vermeulen H. Topical silver for preventing wound infection. Cochrane Database of Systematic Reviews 2010, Issue 3. [DOI: 10.1002/14651858.CD006478.pub2]

Thompson 1999

Thompson SG, Sharp SJ. Explaining heterogeneity in metaanalysis: a comparison of methods. Statistics in Medicine 1999;18:2693-708.

Thompson 2002

Thompson SG, Higgins JPT. How should meta-regression analyses be undertaken and interpreted?. Statistics in Medicine 2002;21:1559-74.

Tierney 2007

Tierney JF, Stewart LA, Ghersi D, Burdett S, Sydes MR. Practical methods for incorporating summary time-to-event data into meta-analysis. Trials 2007;7(8):16.

Trengrove 1996

Trengrove NJ, Stacey MC, McGechie DF, Stingemore NF, Mata S. Qualitative bacteriology and leg ulcer healing. Journal of Wound Care 1996;5:277-80.
Vanderwee 2007

Vanderwee K, Clark M, Dealey C, Gunningberg L, Defloor

T. Pressure ulcer prevalence in Europe: a pilot study. Journal

of Evaluation in Clinical Practice 2007;13(2):227-35.

\section{VanGilder 2009}

VanGilder C, Amlung S, Harrison P, Meyer S. Results of the 2008-2009 International Pressure Ulcer Prevalence Survey and a 3-year, acute care, unit-specific analysis. Ostomy/ Wound Management 2009;55(11):39-45.

\section{Ware 1992}

Ware JE Jr, Sherbourne CD. The MOS 36-item short-form health survey (SF-36). I. Conceptual framework and item selection. Medical Care 1992;30(6):473-483.

\section{Wolcott 2008}

Wolcott RD, Rhoads DD, Dowd SE. Biofilms and chronic wound inflammation. Journal of Wound Care 2008;17(8): 333-41.

\section{WUWHS 2008}

World Union of Wound Healing Societies. Principles of Best Practice. Wound infection in clinical practice: An international consensus. Wound infection in clinical practice. An international consensus. London: MEP Ltd, 2008.

\section{Zhao 2010}

Zhao G, Hiltabidel E, Liu Y, Chen L, Liao Y. A crosssectional descriptive study of pressure ulcer prevalence in a teaching hospital in China. Ostomy and Wound Management 2010;56(3):38-42.

* Indicates the major publication for the study

\section{A P P E N D I C E S}

\section{Appendix I. Risk of bias assessment (individually randomised controlled trials)}

\section{Was the allocation sequence randomly generated?}

\section{Low risk of bias}

The investigators describe a random component in the sequence generation process, such as referring to a random number table; using a computer random number generator; tossing a coin; shuffling cards or envelopes; throwing dice; drawing of lots.

\section{High risk of bias}

The investigators describe a non-random component in the sequence generation process. Usually, the description would involve some systematic, non-random approach, for example, sequence generated by odd or even date of birth; sequence generated by some rule based on date (or day) of admission; sequence generated by some rule based on hospital or clinic record number. 


\section{Unclear risk of bias}

Insufficient information about the sequence generation process provided to permit judgement of low or high risk of bias.

\section{Was the treatment allocation adequately concealed?}

\section{Low risk of bias}

Participants and investigators enrolling participants could not foresee assignment because one of the following, or an equivalent method, was used to conceal allocation: central allocation (including telephone, web-based and pharmacy-controlled randomisation); sequentially numbered drug containers of identical appearance; sequentially numbered, opaque, sealed envelopes.

\section{High risk of bias}

Participants or investigators enrolling participants could possibly foresee assignments and thus introduce selection bias, such as allocation based on using an open random allocation schedule (e.g. a list of random numbers); assignment envelopes were used without appropriate safeguards (e.g. if envelopes were unsealed or non-opaque or were not sequentially numbered); alternation or rotation; date of birth; case record number; any other explicitly unconcealed procedure.

\section{Unclear risk of bias}

Insufficient information provided to permit judgement of low or high risk of bias. This is usually the case if the method of concealment is not described or is not described in sufficient detail to allow a definitive judgement, for example, if the use of assignment envelopes is described, but it remains unclear whether envelopes were sequentially numbered, opaque and sealed.

\section{Blinding (participants, personnel and outcome assessors) - was knowledge of the allocated interventions adequately prevented during the study?}

\section{Low risk of bias}

Any one of the following.

- No blinding, but the review authors judge that the outcome and the outcome measurement are not likely to be influenced by lack of blinding.

- Blinding of participants and key study personnel ensured, and unlikely that the blinding could have been broken.

- Either participants or some key study personnel were not blinded, but outcome assessment was blinded and the non-blinding of others is unlikely to introduce bias.

\section{High risk of bias}

Any one of the following.

- No blinding or incomplete blinding, and the outcome or outcome measurement is likely to be influenced by lack of blinding.

- Blinding of key study participants and personnel attempted, but likely that the blinding could have been broken.

- Either participants or some key study personnel were not blinded, and the non-blinding of others is likely to introduce bias.

\section{Unclear risk of bias}

Either of the following.

- Insufficient information provided to permit judgement of low or high risk of bias.

- The study did not address this outcome. 


\section{Were incomplete outcome data adequately addressed?}

\section{Low risk of bias}

Any one of the following.

- No missing outcome data.

- Reasons for missing outcome data unlikely to be related to true outcome (for survival data, censoring unlikely to be introducing bias).

- Missing outcome data balanced in numbers across intervention groups, with similar reasons for missing data across groups.

- For dichotomous outcome data, the proportion of missing outcomes compared with observed event risk is not enough to have a clinically relevant impact on the intervention effect estimate.

- For continuous outcome data, plausible effect size (difference in means or standardised difference in means) among missing outcomes is not enough to have a clinically relevant impact on observed effect size.

- Missing data have been imputed using appropriate methods.

\section{High risk of bias}

Any one of the following.

- Reason for missing outcome data likely to be related to true outcome, with imbalance in numbers or reasons for missing data across intervention groups.

- For dichotomous outcome data, the proportion of missing outcomes compared with observed event risk is enough to induce clinically relevant bias in intervention effect estimate.

- For continuous outcome data, plausible effect size (difference in means or standardised difference in means) among missing outcomes is enough to induce clinically relevant bias in observed effect size.

- 'As-treated' analysis done with substantial departure of the intervention received from that assigned at randomisation.

- Potentially inappropriate application of simple imputation.

\section{Unclear risk of bias}

Either of the following.

- Insufficient reporting of attrition/exclusions to permit judgement of low or high risk of bias (e.g. number randomised not stated, no reasons for missing data provided).

- The study did not address this outcome.

\section{Are reports of the study free of the suggestion of selective outcome reporting?}

\section{Low risk of bias}

Either of the following.

- The study protocol is available and all of the study's prespecified (primary and secondary) outcomes that are of interest in the review have been reported in the prespecified way.

- The study protocol is not available but it is clear that the published reports include all expected outcomes, including those that were prespecified (convincing text of this nature may be uncommon).

\section{High risk of bias}

Any one of the following.

- Not all of the study's prespecified primary outcomes have been reported.

- One or more primary outcomes are reported using measurements, analysis methods or subsets of the data (e.g. subscales) that were not prespecified.

- One or more reported primary outcomes were not prespecified (unless clear justification for their reporting is provided, such as an unexpected adverse effect).

Antibiotics and antiseptics for pressure ulcers (Protocol)

Copyright @ 2015 The Cochrane Collaboration. Published by John Wiley \& Sons, Ltd. 
- One or more outcomes of interest in the review are reported incompletely so that they cannot be entered in a meta-analysis.

- The study report fails to include results for a key outcome that would be expected to have been reported for such a study.

\section{Unclear risk of bias}

Insufficient information provided to permit judgement of low or high risk of bias. It is likely that the majority of studies will fall into this category.

\section{Other sources of potential bias}

\section{Low risk of bias}

The study appears to be free of other sources of bias.

\section{High risk of bias}

There is at least one important risk of bias. For example, the study:

- had a potential source of bias related to the specific study design used; or

- has been claimed to have been fraudulent; or

- had some other problem.

\section{Unclear risk of bias}

There may be a risk of bias, but there is either:

- insufficient information to assess whether an important risk of bias exists; or

- insufficient rationale or evidence that an identified problem will introduce bias.

\section{Appendix 2. Risk of bias assessment for cluster-randomised controlled trials}

In cluster-randomised trials, particular biases to consider include:

- recruitment bias;

- baseline imbalance;

- loss of clusters;

- incorrect analysis; and

- comparability with individually randomised trials.

Recruitment bias: can occur when individuals are recruited to the trial after the clusters have been randomised, as the knowledge of whether each cluster is an 'intervention' or 'control' cluster could affect the types of participants recruited.

Baseline imbalance: cluster-randomised trials often randomise all clusters at once, so lack of concealment of an allocation sequence should not usually be an issue. However, because small numbers of clusters are randomised, there is a possibility of chance baseline imbalance between the randomised groups, in terms of either the clusters or the individuals. Although this is not a form of bias as such, the risk of baseline differences can be reduced by using stratified or pair-matched randomisation of clusters. Reporting of the baseline comparability of clusters, or statistical adjustment for baseline characteristics, can help reduce concern about the effects of baseline imbalance.

Loss of clusters: occasionally complete clusters are lost from a trial, and have to be omitted from the analysis. Just as for missing outcome data in individually randomised trials, this may lead to bias. In addition, missing outcomes for individuals within clusters may also lead to a risk of bias in cluster randomised trials.

Incorrect analysis: many cluster-randomised trials are analysed by incorrect statistical methods that do not take the clustering into account. Such analyses create a 'unit of analysis error' and produce over-precise results (the standard error of the estimated intervention effect is too small) and P values that are too small. They do not lead to biased estimates of effect. However, if they remain uncorrected, they will receive too much weight in a meta-analysis.

Antibiotics and antiseptics for pressure ulcers (Protocol)

Copyright (๑) 2015 The Cochrane Collaboration. Published by John Wiley \& Sons, Ltd. 
Comparability with individually randomised trials: in a meta-analysis that includes both cluster-randomised and individually randomised trials, or including cluster-randomised trials with different types of clusters, possible differences between the intervention effects being estimated need to be considered. For example, in a vaccine trial of infectious diseases, a vaccine applied to all individuals in a community would be expected to be more effective than a vaccine applied to only half the people. Another example is provided by a Cochrane review of hip protectors (Hahn 2005), where cluster trials showed a large positive effect, whereas individually randomised trials did not show any clear benefit. One possibility is that there was a 'herd effect' in the cluster-randomised trials (which were often performed in nursing homes, where compliance with using the protectors may have been enhanced). In general, such 'contamination' would lead to underestimates of effect. Thus, if an intervention effect is still demonstrated despite contamination in those trials that were not cluster-randomised, a confident conclusion about the presence of an effect can be drawn. However, the size of the effect is likely to be underestimated. Contamination and 'herd effects' may be different for different types of cluster.

\section{WHAT'S NEW}

\begin{tabular}{l|l|l}
\hline Date & Event & Description \\
\hline 20 March 2015 & Amended & COI statement updated Judith Tanner \\
\hline
\end{tabular}

\section{CONTRIBUTIONS OFAUTHORS}

Gill Norman: developed the protocol. Completed the first draft of the protocol and edited the protocol. Approved the final version of the protocol prior to submission and is a guarantor of the protocol.

Jo Dumville: conceived the review question. Developed and co-ordinated the protocol. Secured funding. Performed part of the writing and editing of the protocol. Advised on the protocol, approved the final version prior to submission and is a guarantor of the protocol.

Zena Moore: edited the protocol and performed part of the writing of the protocol. Advised on the protocol.

Judith Tanner: edited the protocol and performed part of the writing of the protocol. Advised on the protocol.

Janice Christie: advised on the protocol, approved the final version prior to submission.

\section{Contributions of editorial base:}

Nicky Cullum: edited the protocol; advised on methodology, interpretation and protocol content. Approved the final protocol prior to submission.

Sally Bell-Syer: coordinated the editorial process. Advised on methodology, interpretation and content. Edited the protocol. Rocio Rodriguez-Lopez: designed the search strategy and edited the search methods section.

\section{DECLARATIONSOF INTEREST}

Gill Norman: nothing to declare.

Jo Dumville: nothing to declare.

Zena Moore: is a member of the medical advisory board of Systagenix Wound Management. The author, Zena Moore, has received an honorarium for speaking at professional meetings for KCI, ConvaTec, Systagenix Wound Management, Fanin Health Care, Molnlycke Health Care and Smith \& Nephew.

Judith Tanner: has received payment for speaking about surgical site infections at study days organised by Smith and Nephew and also Molnlycke Heathcare.

Janice Christie: nothing to declare. 


\section{SOURCES OF SUPPORT}

\section{Internal sources}

- School of Nursing, Midwifery and Social Work, University of Manchester, UK.

\section{External sources}

- National Institute for Health Research, UK.

This report describes independent research funded by the National Institute for Health Research (NIHR Cochrane Programme Grant 13/89/08-High Priority Cochrane Reviews in Wound Prevention and Treatment).

- This project was supported by the National Institute for Health Research, via Cochrane Infrastructure, Cochrane Programme Grant or Cochrane Incentive funding to Cochrane Wounds. The views and opinions expressed therein are those of the authors and do not necessarily reflect those of the Systematic Reviews Programme, NIHR, NHS or the Department of Health, UK. 